\title{
MODELAGEM DO CRESCIMENTO E PRODUÇÃO EMPREGANDO ANATRO EM Pinus taeda L.
}

\author{
GROWTH AND YIELD MODELING OF Pinus taeda L. THROUGH ANATRO
Neumar Irineu Wolff $1 I^{1}$, Andreia Nogueira Bueno ${ }^{2}$, Rodrigo Otávio Veiga de Miranda ${ }^{3}$, Sintia Valerio Kohler ${ }^{4}$, Edson Luis Serpe ${ }^{5}$ \\ 1,2,3 Universidade Estadual do Centro-Oeste, Irati, Paraná, Brasil-neumarwolff@uol.com.br, \\ andias@irati.unicentro.br\&rov_miranda@yahoo.com.br \\ ${ }^{4}$ Universidade Federal do Paraná, Curitiba, Paraná, Brasil-sintiakohler@yahoo.com.br \\ ${ }^{5}$ LWARCEL, Lençóis Paulista, São Paulo, Brasil-serpe.edson@gmail.com
}

RESUMO

\begin{abstract}
A busca do setor florestal por conhecimento de prognoses do crescimento e produção de florestas equiâneas no sul do Brasil é constante. No presente trabalho objetivou-se realizar o ajuste de modelos matemáticos através de dados obtidos pela análise de tronco completa (ANATRO) para gerar as equações que melhor representem o crescimento e a produção futura dos plantios de Pinus taeda. Foram utilizados dados de inventário florestal agrupados em classes de sítio estratificados em classes de diâmetro para a seleção das 144 árvores amostradas para a ANATRO. Foram ajustados dois modelos para a obtenção dos diâmetros com casca nas idades anteriores à coleta. A modelagem do crescimento e produção em nível de povoamento foi realizada através do ajuste de modelos matemáticos. O modelo de Clutter (1963) resultou as melhores projeções da área basal e do volume para o povoamento. Comprovando assim que os resultados da análise de tronco podem ser considerados uma ferramenta apropriada para a modelagem do crescimento e produção.
\end{abstract}

PALAVRAS-CHAVE: Inventário florestal, Prognose, Sítio.

\begin{abstract}
The search of the forestry sector for knowledge of the prognoses of the growth and production of equidistant forests in the south of Brazil is constant. In the present study aimed to carry out the adjustment of mathematical models using data obtained by the complete stem analysis (ANATRO) to generate the equations that best represent the growth and future production of Pinus taeda plantations. We used forest inventory data grouped in site classes stratified in diameter classes to select the 144 trees sampled for ANATRO. Two models were adjusted to obtain the bark diameters at the ages prior to collection. The modeling of growth and seedling level production was performed through the adjustment of mathematical models. The Clutter model (1963) resulted in the best projections of basal area and volume for settlement. Thus, proving that the results of trunk analysis can be considered an appropriate tool for modeling growth and production.
\end{abstract}

KEY WORDS: Forestry inventory, Prognosis, Site. 


\section{INTRODUÇÃO}

A quantificação do crescimento e produção consiste em uma condição essencial para definir a utilização dos bens advindos da floresta, além de fornecer informações que subsidiam a tomada de decisões para muitas atividades do setor. Segundo Daniel \& Yared (1987), o crescimento de um povoamento florestal pode ser conhecido a partir de medições periódicas ou análise de tronco (ANATRO) para espécies com anéis de crescimento visíveis.

Na ANATRO considera-se cada ano de crescimento como uma árvore, permitindo obter um extenso conjunto de dados apropriados para ajustes de equações de volume, implicando em redução dos custos de obtenção de dados comparativamente ao inventário florestal (THOMAS et al., 2006).

A ANATRO convencional, que envolve a coleta de discos das árvores ao longo do fuste e posterior medição dos anéis de crescimento anuais, vem sendo utilizada desde o fim do século XIX e começo do século XX (MACHADO et al., 2013).

Um avanço tecnológico pode ser notado na medição dos anéis, mostrando a tendência da adoção de sistemas que possuam interface eletrônica com computadores, funcionando como gravador e editor de dados (ROSOT, 2002), ou equipamentos específicos como o LINTAB.

A análise de tronco tem sido empregada em trabalhos de modelagem do crescimento e produção, conforme pode ser observado em alguns trabalhos (ELESBÃO, 2008; NOVAES, 2009; HESS \& SCHNEIDER, 2010; SANQUETTA et al., 2010; MUNHOZ, 2011).

A partir das informações obtidas na ANATRO pode-se ajustar modelos matemáticos que permitem obter estimativas para diversas variáveis da floresta, possuindo grande importância na modelagem do crescimento e da produção florestal (CLUTTER et al., 1983).

Estes modelos consagrados na literatura diferem de acordo com o nível de detalhamento, segundo Clutter et al. (1983) podem ser divididos em três tipos de modelos.

I. modelos explícitos ou de povoamento total, que fornecem estimativas da produção por unidade de área a partir de informações relacionados ao povoamento em geral;

II. modelos implícitos ou de classe diamétrica, que estimam a produção considerando a estrutura do povoamento em função da frequência dos diâmetros;

III. modelos de árvores individuais, que avaliam a produção florestal considerando árvores individualmente.

Diante do exposto, o presente trabalho tem por objetivo avaliar o desempenho da utilização de dados oriundos de análise de tronco completa como base para a modelagem do crescimento e da produção de povoamentos florestais de Pinus taeda L.

\section{MATERIAL E MÉTODOS}

\section{Localização da pesquisa}

A pesquisa foi realizada no município de Bituruna, estado do Paraná. De acordo com Köppen, o clima da região é caracterizado como clima temperado ( $\mathrm{Cfb}$ ). A estação meteorológica, apresenta histórico de precipitação média anual de $1.776 \mathrm{~mm}$ e temperatura média de $16,4^{\circ} \mathrm{C}$ (máxima 22,9 e mínima $11,7^{\circ} \mathrm{C}$ ).

\section{Avaliação do sítio}

Os dados inicialmente empregados são compostos por 781 parcelas permanentes, circulares de $600 \mathrm{~m}^{2}$ de área de um inventário realizado em 2010 de $P$. taeda, com idades entre 5 e 18 anos.

Em cada medição, foram registrados a altura total de $20 \%$ das árvores e de seis dominantes e o DAP (diâmetro a altura de 1,3 $\mathrm{m}$ do solo) de todas as árvores das parcelas. Para avaliação da capacidade produtiva, foi utilizado o modelo de Schumacher (CAMPOS \& LEITE, 2009). Na sequência foram construídas três curvas de sítio pelo método da curva guia, com a idade índice de 15 anos.

\section{ANATRO}

Na seleção das árvores para a ANATRO considerou-se a estratificação em classes de diâmetro em cada sítio pelo método de Sturges, visando amostrar árvores que contemplassem a variação diamétrica. Como as distribuições diamétricas apresentaram distribuição normal, definiu-se a amostragem proporcional à frequência, considerando maior número de árvores nas classes mais representativas. Foram medidas 48 árvores em cada classe de sítio, totalizando 144 árvores.

Amostras na forma de discos foram coletadas nas alturas absolutas de 0,05 $\mathrm{m}$ e 1,3 $\mathrm{m}$, e mais nove discos nas alturas relativas a $15,25,35,45,55,65,75,85$ e $95 \%$ da altura total da árvore.

A medição dos anéis de crescimento foi efetuada com o equipamento LINTAB 6.0 software TSAP (Time Series Analysis Program). Os dados obtidos foram processados pelo software FlorExel (ARCE et al., 2002). Com isto obtiveram-se informações da evolução de diâmetro, área 
transversal, altura e volume em cada um dos anos passados de cada árvore, representando seu crescimento.

Foram utilizados e ajustados por regressão linear dois modelos para estimar os diâmetros com casca nas idades passadas em função dos diâmetros sem casca medidos (Tabela 1).

Tabela 1. Modelos empregados para estimativa do diâmetro com casca.

\begin{tabular}{cc}
\hline $\mathbf{n}^{\circ}$ & Modelo \\
\hline 1 & $d_{c c}=\beta_{0}+\beta_{1} \cdot d_{s c}$ \\
2 & $d_{c c}=\beta_{0}+\beta_{1} \cdot d_{s c}+\beta_{2} \cdot d_{s c}^{2}+\beta_{3} . S+\beta_{4} \cdot d_{s c} \cdot I+$ \\
$\beta_{5} \cdot \frac{1}{I}+\beta_{6} . I+\beta_{7} . h t . I$
\end{tabular}

$\overline{\mathrm{dcc}}=$ diâmetro com casca $(\mathrm{cm}) ; \mathrm{dsc}=$ diâmetro sem casca $(\mathrm{cm})$; $\mathrm{S}=$ sítio $(\mathrm{m}) ; \mathrm{I}=$ idade (anos); e ht = altura total (metros).

\section{Estimativas da área basal e volume unitário e por hectare}

Os dados obtidos por ANATRO foram estratificados por sítio, idade e classe diamétricas, e calculada a área basal e o volume médio. Para a extrapolação dos dados para valores médios do povoamento, multiplicou-se pelo número de árvores na idade específica.

\section{Ajuste dos modelos de crescimento e produção}

Campos \& Leite (2009) apontaram que o sistema de equações de Clutter do tipo povoamento total é o mais difundido nas empresas florestais brasileiras, motivo pelo qual foi um dos escolhidos para este ajuste. Assim, foram ajustados também mais dois modelos, um em função da idade e outro em função da idade e do sítio (Tabela 2).

Tabela 2. Modelos de crescimento e produção ajustados.

\begin{tabular}{cc}
\hline $\mathbf{n}^{\mathbf{0}}$ & Modelo \\
\hline (1) & $\ln (\mathrm{V})=\beta_{0}+\frac{\beta_{1}}{I}$ \\
$\operatorname{Schumacher}(1939)$ \\
(2) & $\ln (\mathrm{V})=\beta_{0}+\frac{\beta_{1}}{I \cdot S}$ \\
& $\ln \left(\mathrm{V}_{2}\right)=\beta_{0}+\frac{\beta_{1}}{I_{2}}+\beta_{2} \cdot S+\beta_{3} \cdot \ln \left(G_{2}\right)$ \\
(3) & $\ln \left(G_{2}\right)=\ln \left(G_{1}\right) \cdot\left(\frac{I_{1}}{I_{2}}\right)+\alpha_{0} \cdot\left(1-\frac{I_{1}}{I_{2}}\right)+\alpha_{1} \cdot\left(1-\frac{I_{1}}{I_{2}}\right) \cdot S$ \\
& \\
& Clutter $(1963)$
\end{tabular}

$\mathrm{V}=$ volume $\left(\mathrm{m}^{3} \cdot \mathrm{ha}^{-1}\right), \mathrm{V}_{2}=$ volume $\left(\mathrm{m}^{3} \cdot \mathrm{ha}^{-1}\right)$ na idade $2 ; \mathrm{G}_{1}=$ área basal $\left(\mathrm{m}^{2}\right.$.ha $\left.\mathrm{h}^{-1}\right)$ na idade $1 ; \mathrm{G}_{2}=$ área basal $\left(\mathrm{m}^{2}\right.$.ha-1) na idade 2; $\mathrm{S}=$ índice de sítio $(\mathrm{m}) ; \mathrm{I}=$ idade (anos); $\mathrm{I}_{1}=$ Idade 1 (anos); $\mathrm{I}_{2}=$ Idade 2 (anos); e $\beta \mathrm{i}$ e $\alpha_{\mathrm{i}}=$ parâmetros do modelo.

Os modelos (1) e (2) foram ajustados pelo método dos Mínimos Quadrados Ordinários, e o modelo de Clutter foi ajustado pelo método Mínimo Quadrado em Dois Estágios.
Para o ajuste do modelo de Clutter, foi estimada a área basal inicial em cada classe de sítio.

A partir do modelo com as melhores estatísticas de ajuste, foram construídas as curvas de produção e de incremento médio anual (IMA) e incremento corrente anual (ICA), e por meio das tabelas e gráficos, foi observada a tendência do ponto de vista biológico das estimativas geradas e o comportamento da Idade Técnica de Corte (ITC) com o aumento da capacidade produtiva.

\section{Avaliação dos modelos}

Todos os modelos ajustados foram avaliados considerando as estatísticas coeficiente de determinação ajustado ( $R^{2}{ }_{\text {ajust }}$ ), erro padrão da estimativa relativo ( $S_{y x} \%$ ) e análise gráfica de resíduos.

\section{RESULTADOS E DISCUSSÃO}

\section{Capacidade produtiva e ANATRO}

Os coeficientes e estatísticas do modelo de Schumacher ajustado apresentados na Tabela 3 permitem construir a curva de sítio média. A distribuição gráfica dos resíduos gerados pode ser observada na Figura 1.

Tabela 3. Coeficientes e estatísticas de ajuste do modelo Schumacher para a estimativa de Hdom

\begin{tabular}{ccc}
\hline Modelo & \multicolumn{2}{c}{ Estatísticas } \\
\hline \multirow{2}{*}{$\operatorname{Ln}($ Hdom $)=3,306181-5,423688 .\left(\frac{1}{I}\right)$} & $\mathrm{R}^{2}{ }_{\text {ajust }}$ & 0,72 \\
& $\mathrm{~S}_{\mathrm{yx}}(\mathrm{m})$ & 1,40 \\
& $\mathrm{~S}_{\mathrm{yx}}(\%)$ & 10,3 \\
\hline
\end{tabular}

O modelo apresentou estatísticas dentro da normalidade, estimando a altura dominante sem tendências conforme sua distribuição de resíduos, exceto para idades superiores a 15 anos pela pouca representatividade de parcelas nessas idades.

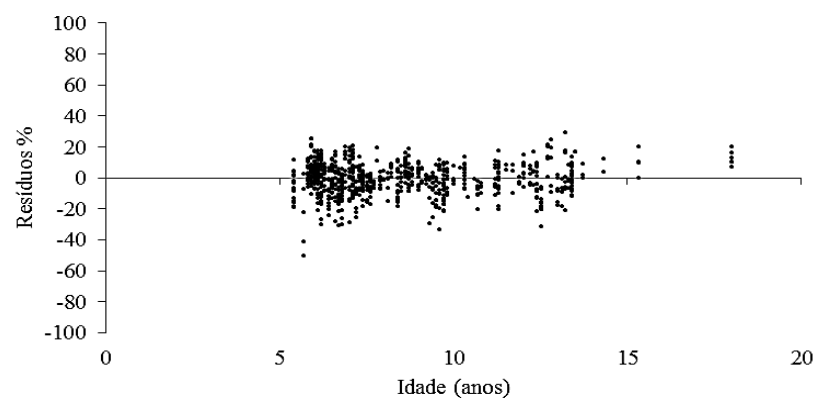

Figura 1. Distribuição de resíduos da estimativa da altura dominante em relação à idade 
A partir da curva guia foram construídas as curvas de sítio, definindo-se três classes de sítio com idade índice de 15 anos. Como resultado a classe de sítio I teve seus limites compreendidos entre 22 a $25 \mathrm{~m}$, a classe de sítio II entre 17 a $21 \mathrm{~m}$ e a classe de sítio III entre 13 a $16 \mathrm{~m}$ (Figura 2), sendo considerado o centro de cada classe na modelagem.

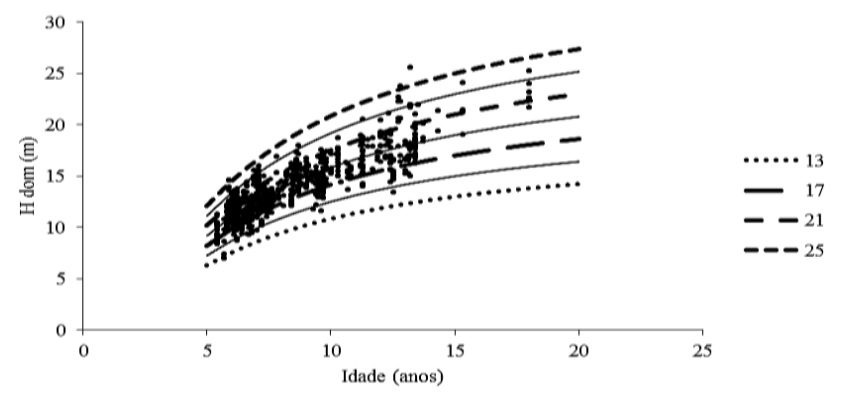

Figura 2. Limite das curvas de sítio.

Como a maioria das parcelas utilizadas para a construção das curvas de sítio encontra-se na classe II, foram selecionadas 44 parcelas desta classe, e 22 parcelas para as classes I e III.

A partir dos dados da ANATRO foram obtidas as estimativas por árvore em cada ano de vida, possibilitando obter estimativas médias do crescimento passado do povoamento.

\section{Estimativa dos diâmetros com casca}

Na Tabela 4 são apresentados os ajustes dos modelos para estimar os diâmetros com casca.

Tabela 4. Modelos ajustados para estimar o diâmetro com casca e suas estatísticas.

\begin{tabular}{ccccc}
\hline $\mathbf{N}^{\circ}$ & Equação ajustada & $\mathbf{R}_{\text {ajust. }}$ & $\begin{array}{c}\mathbf{S}_{\mathbf{y x}} \\
(\mathbf{c m})\end{array}$ & $\begin{array}{c}\mathbf{S}_{\mathbf{y x}} \\
(\mathbf{\%})\end{array}$ \\
\hline (1) & $d_{c c}=0,024184+1,062557 \cdot d_{s c}$ & 0,9983 & 0,38 & 3,14 \\
& $d_{c c}$ & & & \\
& $=-1,73908+1,06751 \cdot d_{s c}$ & & & \\
& $+0,00092 \cdot d_{s c}^{2}-0,01663 \cdot S$ & 0,9986 & 0,35 & 2,90 \\
& $-0,00216 \cdot d_{s c} \cdot I+8,14484 \cdot \frac{1}{I}$ & & & \\
& $+0,17114 \cdot I-0,00311 \cdot h t \cdot I$
\end{tabular}

A distribuição gráfica dos resíduos (Figuras 3) apresenta tendenciosidade nos menores diâmetros. Como este segmento possui baixa representatividade tanto em área basal quanto em volume, esta tendência não prejudica os resultados. Para os demais diâmetros, a distribuição ficou dentro de um limite de $20 \%$ em relação aos valores observados.

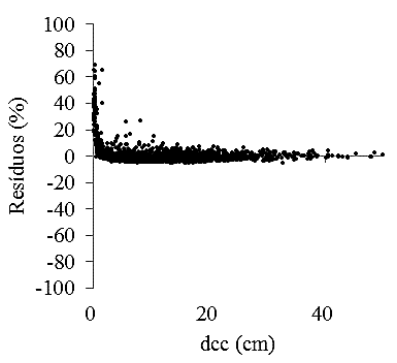

(a)

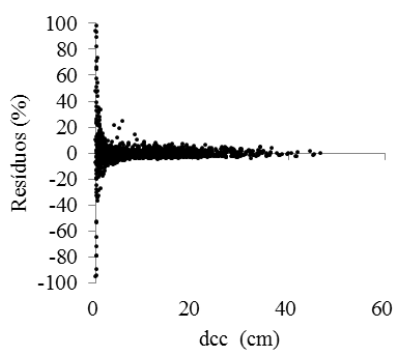

(b)
Figura 3. Distribuição de resíduos do modelo (a) e (b).

\section{Estimativas da área basal e volume unitário e por hectare}

Os resultados da ANATRO foram compilados em tabelas que permitiram a obtenção das estimativas individuais médias da área transversal e do volume por sítio.

\section{Ajuste dos modelos de crescimento e produção}

Os resultados obtidos nos ajustes e as respectivas equações com seus coeficientes são apresentados na Tabela 5.

As estatísticas de ajuste demonstram melhores resultados para o modelo de Clutter (III), pois apresentou o maior valor do coeficiente de determinação ajustado e os menores erros. Na Figura 4, foram geradas as distribuições dos resíduos do volume para os modelos I e II.

Tabela 5. Modelos ajustados para estimar por sítio o crescimento e produção e suas estatísticas.

\begin{tabular}{|c|c|c|c|c|c|}
\hline$n^{\circ}$ & Sítio & Equação ajustada & $\mathbf{R}^{\mathbf{2}}$ ajust. & $\begin{array}{c}\mathrm{S}_{\mathrm{yx}} \\
\mathrm{m}^{3} \mathrm{ha}^{-1}\end{array}$ & $\mathrm{~S}_{\mathrm{yx}} \%$ \\
\hline \multirow{3}{*}{ (1) } & 1 & $\ln (V)=7,0760+\frac{-17,1990}{I}$ & 0,8284 & 43,734 & 33,67 \\
\hline & II & $\ln (V)=7,0333+\frac{-17,8264}{I}$ & 0,8437 & 29,932 & 31,33 \\
\hline & III & $\ln (V)=7,2032+\frac{-18,9810}{I}$ & 0,9056 & 22,797 & 25,54 \\
\hline (2) & I, II, III & $\ln (V)=6,5783+\frac{-269,6945}{I . S}$ & 0,5894 & 46,798 & 45,24 \\
\hline \multirow{3}{*}{ (3) } & \multirow{3}{*}{ I, II, III } & $\begin{array}{c}\ln \left(V_{2}\right)=3,3097+\frac{-9,3418}{I_{2}}+ \\
0,0134 \cdot S+0,7811 \cdot \ln \left(G_{2}\right)\end{array}$ & 0,9779 & 15,23 & 10,66 \\
\hline & & $\ln \left(G_{2}\right)=\ln \left(G_{1}\right) \cdot\left(\frac{I_{1}}{I_{2}}\right)+$ & & & \\
\hline & & $\begin{array}{c}4,8519 \cdot\left(1-\frac{I_{1}}{I_{2}}\right)+0,0070 \cdot(1- \\
\left.\frac{I_{1}}{I_{2}}\right) \cdot S\end{array}$ & 0,9769 & 1,807 & 7,67 \\
\hline
\end{tabular}

A distribuição gráfica dos resíduos apresentou em sua maioria valores abaixo de $50 \%$ de erro. Nos dois primeiros gráficos referentes aos sítios I e II, não nota nenhuma tendência nos valores estimados, e no sítio III os dados aparentam valores subestimados nas idades iniciais, passando a uma superestimação nas idades médias e 
novamente subestimados nas idades avançadas.

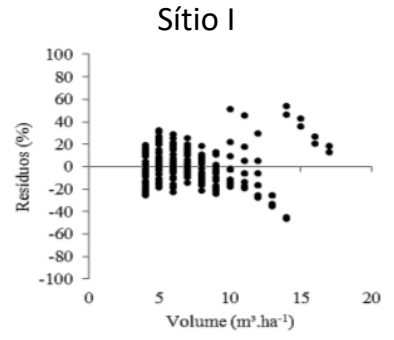

Sítio III

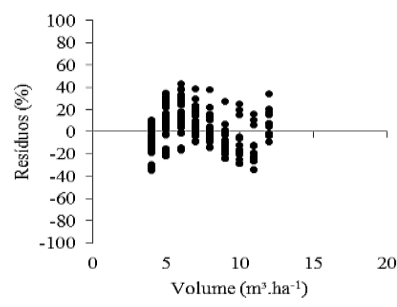

Sítio II

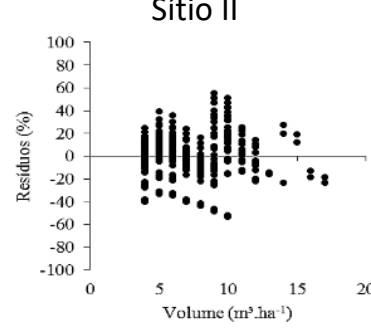

Modelo $f(I, S)$

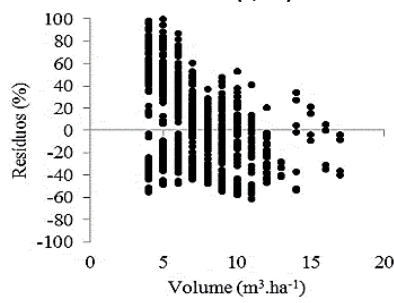

Figura 4. Distribuição dos resíduos para volume do modelo de Schumacher para o Sítio I, Sítio II, Sítio III e para o modelo $\mathrm{f}(\mathrm{I}, \mathrm{S})$.

Na Figura 5 podem ser observadas as distribuições de resíduos para área basal e volume para o modelo de Clutter. Os resíduos ficaram praticamente distribuídos dentro de um intervalo de $20 \%$ em relação à média observada sem tendência aparente.
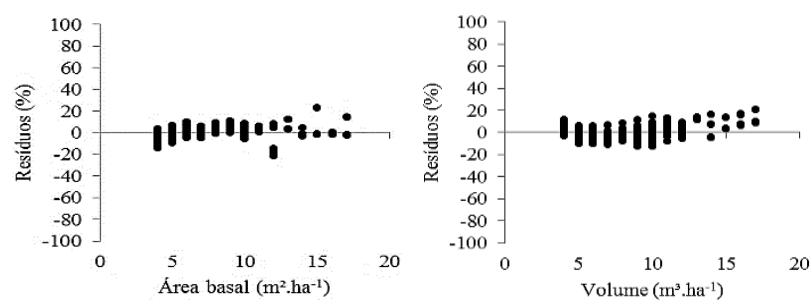

Figura 5. Distribuição dos resíduos para a área basal e para o volume pelo modelo de Clutter.

Foram plotados gráficos com as curvas de produção referentes à área basal e ao volume (Figuras 6 e 7), com as maiores produções no Sítio I e as menores no Sítio III, comprovando a realidade biológica dos ajustes.

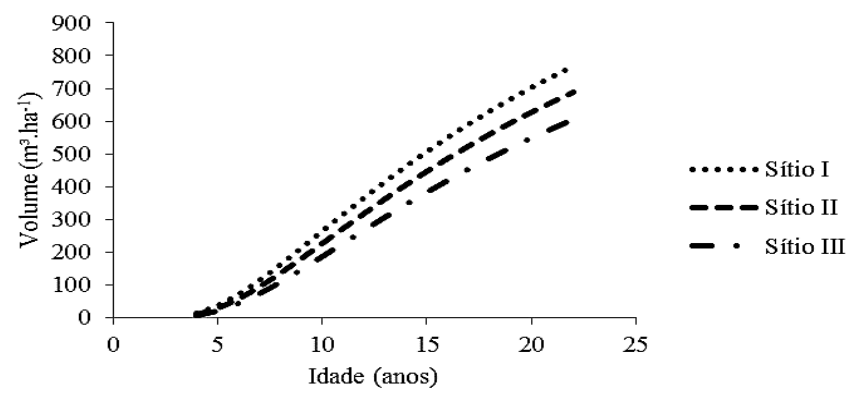

Figura 6. Curvas de produção em volume para os três sítios considerados.

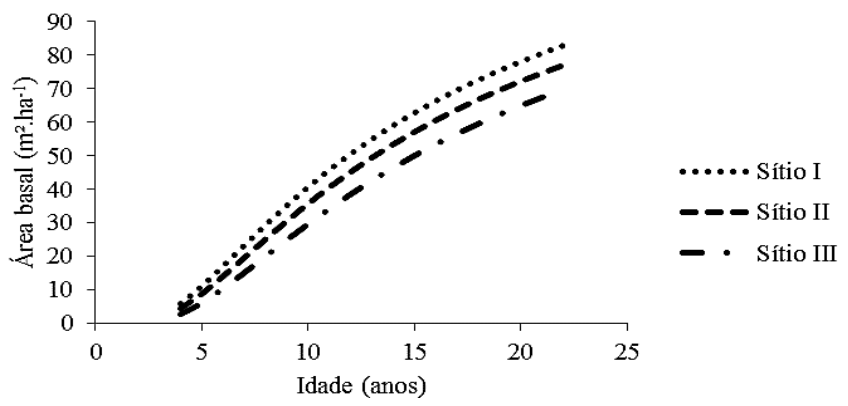

Figura 7. Curvas de produção em área basal para os três sítios considerados.

Nas Figuras 8 e 9 são apresentadas as curvas de incremento corrente anual e incremento médio anual para volume e área basal por classe de sítio.

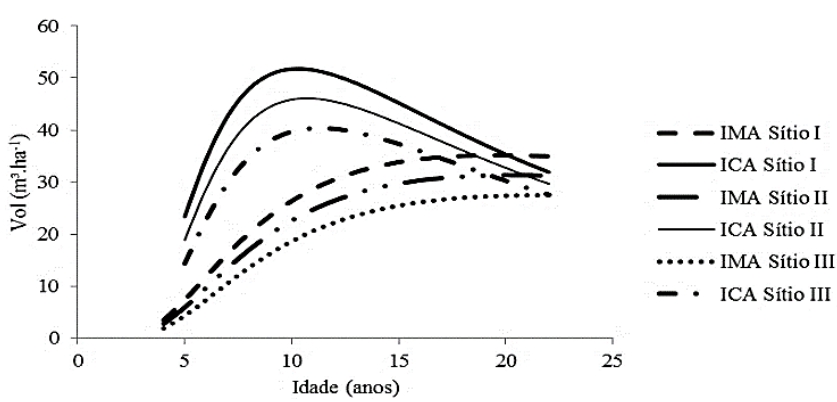

Figura 8. Curvas de incremento médio e corrente anual em volume por sítio.

Conforme os gráficos de produção e as curvas de incremento em volume, pode-se observar que as idades técnicas de corte (ITC), são de 20, 21 e 22 anos para os sítios I, II e III, comprovando que quanto maior o índice de sítio, menor a idade de intervenção.

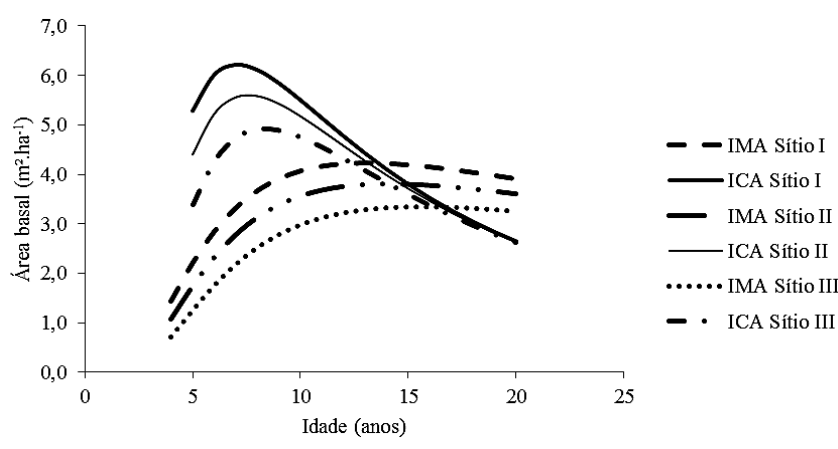

Figura 9. Curvas de incremento médio e corrente anual em área basal por sítio.

Para a área basal, os pontos de cruzamento das curvas ocorrem antes se comparado ao volume, aos 13, 14 e 16 anos, para os sítios I, II e III respectivamente.

Como validação, foram plotados dados de 150 parcelas de inventário não consideradas na ANATRO comparativamente às curvas de produção (Figura 10). 


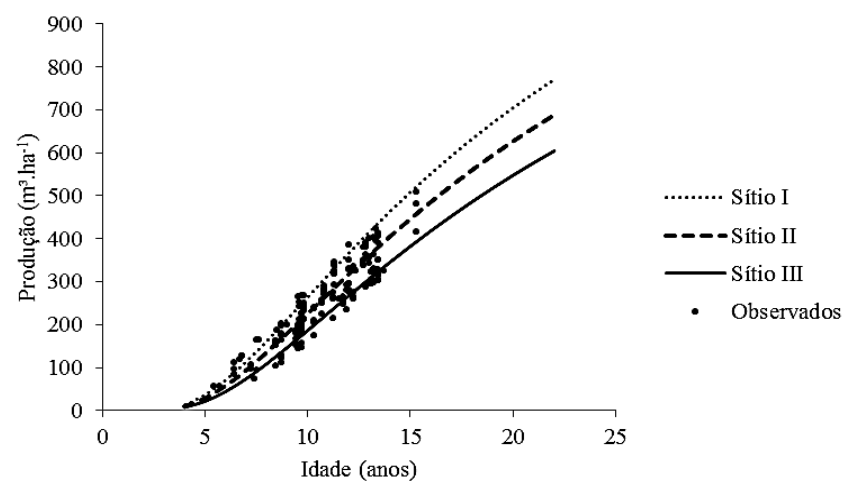

Figura 10. Validação dos resultados - Produção estimada $x$ Produção observada

Conforme a Figura 10, os dados de validação situaramse, em sua maioria, dentro do limite compreendido para os três sítios considerados. Isto comprova que as curvas de produção obtidas podem ser utilizadas para projeções futuras, desde que sejam considerados os volumes suprimidos em eventuais desbastes realizados ao longo dos anos.

\section{CONCLUSÕES}

O modelo de Schumacher foi adequado para representar o índice de sítio para a espécie $P$. taeda no local estudado.

Os modelos de crescimento e produção que utilizam o sítio como variável independente, apresentaram idades técnicas de corte coerentes do ponto de vista biológico, ou seja, para sítios melhores, a idade técnica de corte foi menor.

O modelo de Clutter mostrou-se o mais adequado na obtenção das estimativas de volume e área basal, fornecendo valores coerentes do ponto de vista estatístico e biológico.

Obtiveram-se resultados adequados no emprego da análise de tronco completa como base de dados para estudos de modelagem do crescimento e da produção.

\section{AGRADECIMENTOS}

À Remasa Reflorestadora S.A., pela autorização e apoio no trabalho de campo.

\section{REFERÊNCIAS}

ARCE, J.E. et al. Florexel - Funções florestais desenvolvidas para o Microsoft Excel ${ }^{\odot}$. Curitiba: Centro de Ciências Florestais e da Madeira-CCFM, Universidade Federal do Paraná, 2002. Software (suplemento).

CAMPOS, J.C.C.; LEITE, H.G.; Mensuração Florestal: perguntas e respostas. 3.ed. Viçosa: Editora UFV, 2009.

CLUTTER, J.C. et al. Timber management: a quantitative approach. 3.ed. New York: John Willey, 1983.

DANIEL, O.; YARED, J.A.G. Procedimentos para análise de tronco de espécies florestais. Belém: FCAP, Serviço de Documentação e Informação, 1987.

ELESBÃO, L.E.G. Crescimento e produção de Pinus taeda L. na região dos campos de cima da serra, Rio Grande do Sul. 2008. 73p. (Dissertação de mestrado).

HESS, A.F.; SCHNEIDER, P.R. Crescimento em volume de Araucaria angustifolia (Bertol.) Kuntze em três regiões do Rio Grande do Sul, Brasil. Ciência Florestal, v.20, n.1, p.107-122, 2010.

MACHADO, S.A. et al. Comparação entre análise de tronco digital e convencional em árvores de Mimosa scabrella Bentham e Pinus taeda L. Árvore, v.37, n.2, p.329-337, 2013.

MUNHOZ, J.S.B. Caracterização da produtividade florestal e dos padrões de crescimento de Pinus taeda L. no sul do Brasil através de análise de tronco. 2011. 116p. (Dissertação de mestrado).

NOVAES, D.M. Modelagem do crescimento e produção de povoamentos de Tectona grandis L.f. a partir de análise de tronco. 2009. 52p. (Dissertação de mestrado).

ROSOT, M.A.D. Processamento digital de imagens aplicado à medição de anéis de crescimento na análise de tronco. 2002. 206p. (Tese de doutorado).

SANQUETTA, C.R. et al. Equações individuais e determinação de classes de crescimento para Nectandra grandiflora Nees a partir de dados de análise de tronco. Scientia Agraria, v.11, n.1, p.1-8, 2010.

THOMAS, C. et al. Comparação de equações volumétricas ajustadas com dados de cubagem e análise de tronco. Ciência Florestal, v.16, n.3, p.319-327, 2006. 